\title{
Diacronie
}

Studi di Storia Contemporanea

$N^{\circ} 25,1 \mid 2016$

"Se creare è definire"

\section{Comunicazione politica, informazione e censura a Venezia nella prima età moderna}

\section{Silvia Manzi}

\section{(2) OpenEdition}

Journals

\section{Edizione digitale}

URL: http://journals.openedition.org/diacronie/3881

DOI: $10.4000 /$ diacronie.3881

ISSN: 2038-0925

\section{Editore}

Association culturelle Diacronie

\section{Notizia bibliografica digitale}

Silvia Manzi, «Comunicazione politica, informazione e censura a Venezia nella prima età moderna », Diacronie [Online], N²5, 1 | 2016, documento 10, Messo online il 29 mars 2016, consultato il 24 septembre 2020. URL : http://journals.openedition.org/diacronie/3881 ; DOI : https://doi.org/10.4000/ diacronie.3881 


\section{Comunicazione politica, informazione e} censura a Venezia nella prima età moderna RECENSIONI:

Filippo DE VIVO, Patrizi, informatori, barbieri. Politica e comunicazione a Venezia, Milano, Feltrinelli, 2012, 466 pp.

Mario INFELISE, I padroni dei libri. Il controllo sulla stampa nella prima età moderna, Roma-Bari, Laterza, 2014, 226 pp.

a cura di Silvia MANZI *

Repubblica cosmopolita, snodo di commerci, crocevia di notizie, capitale editoriale, nel XVI e XVII secolo Venezia è un osservatorio privilegiato per un'indagine sulla comunicazione politica, sull'informazione e sulla censura nella prima età moderna. E lo diventa ancora di più durante la vicenda dell'Interdetto, quando la mole di documenti sulla "guerra delle scritture" facilita lo studio sia dei mezzi di comunicazione delle informazioni sia dei controlli esercitati su di esse, come mostrano i recenti libri di Filippo de Vivo ${ }^{1}$ e di Mario Infelise ${ }^{2}$.

Sul primo aspetto si sofferma la ricerca di de Vivo, ricerca che si situa nel solco degli studi sulla formazione dell'opinione pubblica e sulla costruzione dello Stato, inaugurati da Jürgen Habermas ${ }^{3}$, il cui paradigma a partire dagli anni Novanta è stato

\footnotetext{
${ }^{1}$ DE VIVO, Filippo, Patrizi, Informatori, Barbieri. Politica e comunicazione a Venezia nella prima età moderna, Milano, Feltrinelli, 2012.

2 INFELISE, Mario, I padroni dei libri. Il controllo sulla stampa nella prima età moderna, Roma-Bari, Laterza, 2014.

3 HABERMAS, Jurgen, Strukturwandel der Öffentlichkeit, Neuwied, Hermann Luchterhand Verlag, 1962.
} 
progressivamente decostruito dalla storiografia ${ }^{4}$. Paradigma che de Vivo declina da un'angolazione diversa, ossia quella dei modi, dei mezzi, dei luoghi attraverso i quali si comunicano, si diffondono, si recepiscono e si modificano le notizie.

L'analisi storiografica tradizionale incentrata sulla contrapposizione tra autorità secolare e clericale durante l'Interdetto e i più recenti studi ${ }^{5}$ sui compromessi tra autorità secolare ed ecclesiastica sono rivisitati e sviluppati attraverso un'indagine più approfondita rispetto alla precedente edizione inglese di de Vivo dal titolo Information \& Communication in Venice. Rethinking Early Modern Politics ${ }^{6}-$ sugli ambienti, i personaggi, le forme comunicative dello scontro, secondo un modello di «histoire à part entière» 7 .

Ne risulta una ricerca che si pone all'incrocio tra diverse aree disciplinari: la storia urbana, la storia della comunicazione, la storia della lettura e del libro. Proprio sulla scia della storia del libro, come sottolinea de Vivo nell'Introduzione, si è infatti affermata la storia dell'informazione per l'Italia della prima età moderna, storia che ha incentivato studi sulla comunicazione concernenti anche l'alfabetizzazione e i livelli sociali dei lettori.

Pur muovendo da questi studi, de Vivo adotta un diverso approccio metodologico:«ridurre la scala cronologica e geografica, concentrarsi su una singola città » e così «prendere in esame tutti i diversi mezzi di comunicazione verbale studiati finora separatamente e mostrare come interagissero gli uni agli altri $>^{8}$. Ne risulta una suggestiva ricostruzione dei contatti e degli scambi tra i diversi strati sociali in una microsocietà urbana, nella consapevolezza che studiare l'Interdetto soltanto sotto il profilo dell'opposizione tra comunicazione dall'alto e comunicazione dal basso non

\footnotetext{
4 Sul ripensamento del paradigma habermasiano, cfr. DERRIDA, Jacques, L'autre cap, Paris, Les Éditions de Minuit, 1991 [trad. it., Oggi L’Europa, Milano, Garzanti, 1991]; CALHOUN, Craig (edited by), Habermas and the Public Sphere, Boston, M.I.T. Press, 1992; LAKE, Peter, PINCUS, Steve, «Rethinking the Public Sphere in Early Modern England», in Journal of British Studies, 45, 2006, pp. 270-292; BENIGNO, Franco, «Nascita dell'opinione pubblica: Benigno legge Landi», in Storica, XIII, 37, 2007, pp. 175-182; ROSPOCHER, Massimo (edited by), Beyond the Public Sphere. Opinions, Publics, Spaces in Early Modern Europe, Bologna-Berlin, Il Mulino-Duncker\&Humblot, 2012; ROSPOCHER, Massimo, SALZBERG, Rosa, "El vulgo zanza": spazi, pubblici, voci a Venezia durante le guerre d'Italia», in Storica, XVI, 48, 2010, pp. 83-120.

5 DEL COL, Andrea, «L'Inquisizione romana e il potere politico nella repubblica di Venezia (1540-1560)», in Critica Storica, 28, 1991, pp. 189-250; SIGNOROTTO, Giovanni Vittorio, «Venezia e il ritorno dei Gesuiti (1606-1657)», in Rivista di Storia e Letteratura Religiosa, 28, 1992, pp. 277-317; BONORA, Elena, I conflitti della Controriforma: Santità e obbedienza nell'esperienza religiosa dei primi Barnabiti, Firenze, Le Lettere, 1998.

6 DE VIVO, Filippo, Information \& Communication in Venice. Rethinking Early Modern Politics, Oxford, Oxford University Press, 2007.

7 NICCOLI, Ottavia, CAVARZERE, Marco, «A proposito di "Patrizi, Informatori, Barbieri" di Filippo de Vivo», in Quaderni Storici, 144, 2013, pp. 889-902, p. 889.

8 DE VIVO, Filippo, Patrizi, Informatori, Barbieri, cit, p. 24.
} 
avrebbe, infatti, consentito di cogliere a pieno l'eccezionalità del momento storico. Momento storico in cui la "comunicazione" - termine che nel vocabolario politico veneziano designa una ristretta circolazione di notizie all'interno del patriziato diventa "pubblicazione", cioè diffusione incontrollata, prima tra gli informatori professionisti, poi tra più ampi strati della popolazione.

Di qui la presa di distanza dal modello foucaultiano, secondo cui ogni enunciato è «uno strumento di dominio» e i presupposti della comunicazione sono «irrimediabilmente compromessi dal potere ${ }^{9}$, ma in una certa misura anche dal modello opposto - l'idealizzata ed egualitaria sfera pubblica habermasiana - qui applicato a una realtà ben più sfaccettata, in cui la comunicazione politica è interazione tra livelli distinti: i patrizi, gli informatori e i barbieri del titolo.

A questi tre soggetti sono associati tre luoghi-simbolo della città: ai patrizi Palazzo Ducale; agli informatori professionisti il broglio e i ridotti dell'arena politica; ai mercanti, agli artigiani e ai barbieri il ponte di Rialto. Sensibile agli aspetti della cultura materiale, de Vivo disegna, infatti, una mappa dettagliata degli spazi interni ed esterni: le librerie e le botteghe, luoghi di ritrovo che anticipano i "caffè" habermasiani; le calli e, soprattutto, Piazza San Marco. Cuore della topografia veneziana, cuore civico e religioso della Repubblica, teatro dei rituali delle autorità, nelle pagine di de Vivo la piazza è lo "spazio praticato" di de Certeau.

È il luogo privilegiato dove si verifica il trasferimento di notizie - intenzionale e/o accidentale - dagli organi di governo, alla sfera degli informatori, al "popolo". Un modello di "comunicazione triangolare" lo ha definito de Vivo nel più recente saggio Sfera pubblica o triangolo della comunicazione? Informazione e politica nella prima età moderna, apparso non a caso in una raccolta di saggi sulla sfera pubblica curata da Massimo Rospocher ${ }^{10}$. In base a questo modello che, a differenza di quelli di Foucault e di Habermas, non enfatizza il ruolo di un singolo agente - le autorità o il pubblico - e che inoltre evidenzia gli elementi di contatto e di conflitto, componenti fondamentali della comunicazione politica, ai gruppi posti ai tre vertici del triangolo è associato un diverso livello di accesso alle informazioni.

Considerando le modalità d'espressione dei tre poli, il testo di de Vivo diventa un "palinsesto" che dietro le fonti ufficiali (carte delle autorità statali o corrispondenza tra istituzioni) o semiufficiali (lettere private, manoscritti che circolano in forma clandestina, avvisi compilati dai reportisti) cela le tracce di quella comunicazione orale che sfugge a ogni forma di controllo, come le chiacchiere dei barbieri, "Menocchi

\footnotetext{
${ }^{9}$ Ibidem, p. 31 .

10 DE VIVO, Filippo, Public Sphere or Communication Triangle? Information and Politics in Early Modern Europe, in ROSPOCHER, Massimo (a cura di), op. cit., pp. 115-136.
} 
lagunari”, che collocano l'autore nel solco della microstoria culturale di Burke e di Ginzburg. L'attenzione nei confronti dell'oralità, se da un canto gli consente di superare la dicotomia manoscritto-stampa, dall'altro lo costringe a confrontarsi con gli inevitabili limiti che essa impone e che diventano stringenti se le voci da salvare dall'oblio sono quelle di chi, pur non occupando i vertici della gerarchia sociale, grazie alla struttura fluida delle reti comunicative, ha comunque accesso, prim'ancora della loro diffusione ufficiale, a informazioni tradizionalmente precluse.

Proprio al fine di zittire i rumores della piazza, delle calli, delle botteghe, si ricorre alla "ufficializzazione" del conflitto attraverso "scritture" riprodotte in centinaia di esemplari di diversa lunghezza, lingua e formato. De Vivo registra in appendice ben 155 titoli pubblicati in 321 edizioni, vale a dire più del doppio rispetto alle precedenti congetture. Ł̀ una campagna di stampa che, come sottolinea l'autore, non ha precedenti: gli scontri con la Santa Sede del 1483 e del 1509 non ebbero la risonanza del conflitto dell'Interdetto, quando le magistrature scendono in campo - fatto eccezionale in Antico Regime - per allargare il dibattito a cerchie più ampie di fruitori dell'informazione.

Una novità a cui Venezia, che inizialmente aveva negato l'esistenza dell'Interdetto, fedele alla tradizionale strategia del "diniego", è costretta ad arrendersi per controbattere agli attacchi a stampa degli autorevoli esponenti delle gerarchie ecclesiastiche: i cardinali Cesare Baronio e Roberto Bellarmino. Ma novità su cui de Vivo invita a riflettere è anche l'uso del volgare da parte delle autorità coinvolte in una contesa in cui, come sottolinea Paolo Sarpi, le parole sono armi. Scelta giustificata da Bellarmino quando, nella Risposta al trattato dei sette teologi di Venezia sopra l'Interdetto, adduce la "necessità della Chiesa" di difendersi dalla panflettistica degli avversari usando il loro stesso idioma ${ }^{11}$. Se scopo dell'Interdetto è far cedere la Repubblica, aizzando il popolo, ora agente politico riconosciuto, il volgare diventa risorsa indispensabile: l'obiettivo di un'ampia risonanza del conflitto e la garanzia che a chiunque fosse nota la scomunica collettiva che avrebbe impedito la somministrazione dei sacramenti e posto Venezia fuori dalla Chiesa deve scontare il prezzo della

\footnotetext{
${ }^{11} \ll$ Sono alcuni che si meravigliano che io risponda a certi libretti volgari che paiono - e sono veramente di poca sostanza e di meno dottrina - stimando che in questo si avvilisca la dignità delle opere latine che in altri tempi ho dato alla stampa. A questi tali rispondo, che a far ciò mi ha mosso l'esempio de' maggiori, la necessità della Chiesa, e l'importunità degli avversarii [...] E sebbene questi libretti sono piccoli e volgari, nondimeno non è piccolo il danno che possono fare nell'anime de' fedeli, se si lasciano andare senza risposta», cfr. FRAGNITO, Gigliola, Proibito capire. La Chiesa e il volgare nella prima età moderna, Bologna, Il Mulino, 2005, p. 186 (cit. in GODMAN, Peter, The Saint as Censor. Robert Bellarminebetween Inquisition and Index, Leiden, Brill, 2000, p. 191).
} 
"detronizzazione" del latino ${ }^{12}$. Ecco allora giustificata la scelta del volgare per il Breue di censvre et interdetto di Paolo V e per il "protesto"redatto da Paolo Sarpi, consultore giuridico della Repubblica. Stessa lingua, stesse strategie comunicative: il protesto viene affisso sulle porte delle chiese di cui il monitorio papale ordina la chiusura. Identici i destinatari: gli ecclesiastici veneziani e, attraverso loro, i fedeli.

Approfondendo le dinamiche della comunicazione e della diffusione di notizie, de Vivo rileva che le informazioni riservate - sulle decisioni dei Consigli o sui dispacci degli ambasciatori - approdano sulla bocca dei frequentatori di mercati e "barberie" sotto forma di brandelli di discussioni, destinati a ricomporsi in un pubblico dibattito che spesso trasfigura gli eventi politici e le dispute internazionali.

La vicenda dell'Interdetto può essere allora considerata come l'«eccezionalenormale» ${ }^{13}$ nella Venezia del Seicento dove, soprattutto nella seconda metà del secolo, l'incremento delle gazzette con ragguagli su eventi europei moltiplica i fatti su cui discutere ${ }^{14}$.

Negli spazi pubblici si intrecciano argomenti politici e religiosi. Non sempre si tratta di discorsi coerenti, ma di accostamenti di frammenti di varia provenienza che compongono il mosaico di una filosofia composita, continuamente rimodellata durante le conversazioni. Sono discorsi che non si limitano a recepire supinamente notizie calate dall'alto, ma le filtrano ed elaborano fino a giungere a criticare le decisioni politiche e il magistero della Chiesa. Ciò si evince in maniera efficace dalla ricostruzione di Federico Barbierato dei percorsi della miscredenza in ambiente veneziano tra XVII e XVIII secolo, che mostrano la stretta relazione tra lo sviluppo del mercato dell'informazione e la nascita di un embrionale senso critico tradottosi in un atteggiamento scettico nei confronti della politica e della religione. La lettura delle gazzette induce, infatti, a interrogarsi sugli arcana imperii e a mettere in discussione l'aura di sacralità che avvolge la religione. La miscredenza si insinua così nelle crepe sfuggite alla vigilanza della Chiesa, investendo temi come l'esistenza del paradiso o dell'inferno, in sintonia con la teoria dell'impostura politica delle religioni ${ }^{15}$.

Parte integrante della comunicazione - e di qui la pertinenza di rendere conto dei due libri insieme - è il controllo della medesima. L’industria tipografica a Venezia, almeno fino alla peste seicentesca, è ancora molto attiva ed è necessariamente oggetto

\footnotetext{
${ }^{12}$ L'espressione è di WAQUET, Françoise, Le latin ou l'empire d'un signe. $X V I^{e}-X X^{e}$ siècle, Paris, Albin Michel, 1998, p. 59.

${ }^{13}$ GRENDI, Edoardo, «Micro-analisi e storia sociale», in Quaderni storici, 35, 1977, pp. 506-520, p. 512.

${ }^{14}$ Su questi temi, cfr. INFELISE, Mario, Prima dei giornali. Alle origini della pubblica informazione, Roma-Bari, Laterza, 2005.

${ }^{15}$ BARBIERATO, Federico, Politici e ateisti. Percorsi della miscredenza a Venezia fra Sei e Settecento, Milano, Unicopli, 2006.
} 
di vigilanza. Ne I padroni dei libri Mario Infelise si sofferma sulle tematiche relative alla censura fino ad ora maggiormente esplorate. Per quanto riguarda l'Europa la ricerca si sarebbe concentrata sulle mire assolutistiche dei principi, finalizzate a controllare i sudditi e a plasmare le loro opinioni; mentre al centro degli studi sull'Italia sarebbero state le pratiche repressive avviate durante la lunga stagione della Controriforma, responsabili del declino della cultura italiana e del suo isolamento da quella europea.

Dopo i pioneristici studi degli anni Settanta ${ }^{16}$, l'apertura degli archivi della Congregazione per la Dottrina della Fede nel 1998 ha suscitato inevitabilmente una nuova attenzione per la censura ecclesiastica e prodotto una messe di studi ${ }^{17}$ che hanno permesso di correggere l'immagine monolitica degli apparati preposti alla censura, «mostrando il significato degli attori e delle logiche di potere, spesso conflittuali, che ne determinano gli orientamenti» ${ }^{18}$. La ricerca di Infelise, in linea con l'interesse attuale per l'evoluzione delle forme di controllo della comunicazione e per la modalità di costruzione dell'odierno concetto di libertà d'espressione ${ }^{19}$, si propone di recuperare il ruolo delle autorità statali nel controllo della comunicazione e di ridimensionare quello attribuito alla Chiesa. Come il precedente lavoro I libri probiti2o, I padroni dei libri segna così «la definitiva laicizzazione degli studi sulla censura, il loro irreversibile affrancamento da schemi e preoccupazioni riconducibili, nella storiografia italiana, allo scontro ideologico fra Stato e Chiesa». Ne deriva un nuovo approccio storiografico, in cui la censura è «un oggetto storiografico maturo e per certi aspetti nuovo, che si avvale del contributo decisivo di discipline relativamente giovani e in crescita quali la storia dell'editoria e della lettura ${ }^{21}$

\footnotetext{
${ }^{16}$ Tra i primi a intuire i fondamentali sviluppi del filone degli studi della censura ecclesiastica per la storia e cultura europea, cfr. ROTONDÓ, Antonio, La censura ecclesiastica e la cultura, in Storia d'Italia, vol. V, I Documenti, Torino, Einaudi, 1973, pp. 1397-1492. Tra i lavori pioneristici degli anni Settanta cfr. anche TEDESCHI, John, Florentine documents for a history of the "Index prohibited books", in MOLHO, Anthony, TEDESCHI, John (edited by), Renaissance studies in honor of Hans Baron, Firenze, Sansoni, 1971, pp. 577-605; LOPEZ, Pasquale, Inquisizione stampa e censura nel Regno di Napoli tra '500 e '600, Napoli, Edizioni del Delfino,1974; GRENDLER, Paul, The Roman Inquisition and the Venetian Press,1540-1605, Princeton, Princeton University Press, 1977; DE FREDE, Carlo, Ricerche per la storia della stampa e la diffusione delle idee riformate nell'Italia del Cinquecento, Napoli, De Simone, 1985.

17 FRAGNITO, Gigliola, Gli studi sulla censura ecclesiastica nella prima età moderna: bilanci e prospettive, in GULIA, Luigi, HERKLOTZ Ingo, ZEN Stefano (a cura di), Società, cultura e vita religiosa in età moderna. Studi in onore di Romeo De Maio, Sora, Centro di Studi Sorani "Vincenzo Patriarca", 2009, pp. 163-176.

${ }^{18}$ LANDI, Sandro, Stampa, censura e opinione pubblica in età moderna, Bologna, Il Mulino, 2011, p. 78.

${ }^{19}$ Cfr. CAVARZERE, Marco, La prassi della censura nell'Italia del Seicento, Roma, Edizioni di Storia e Letteratura, 2011; TORTAROLO, Edoardo, L'invenzione della libertà. Censura e libertà nel Settecento, Roma, Carocci, 2011.

${ }^{20}$ INFELISE, Mario, I libri proibiti. Da Gutenberg all'Encyclopédie, Roma-Bari, Laterza,1999.

${ }^{21}$ LANDI, Sandro, «Censure - Landi legge Infelise», in VIII, Storica, 22, 2002, p. 207.
} 
Rispetto al lavoro precedente, Infelise indaga un periodo più limitato e un preciso contesto politico, quello veneziano alla fine del Cinquecento e nel corso del Seicento, vale a dire un periodo in cui l'Interdetto è solo una tra le tante occasioni di scontro tra Stato e Chiesa per il controllo della comunicazione e della stampa. Meno interessato alle modalità di applicazione delle norme censorie emanate da Roma, esamina le interferenze tra i due poteri, le tensioni che ne derivano e che sfociano in un alternarsi di estenuanti guerre di posizione e in aperti conflitti, quali quelli, appunto, dell'Interdetto e della guerra di Castro (1640-44). A monte l'irrisolta difficoltà per entrambe le parti di elaborare e perseguire politiche coerenti, di definire con chiarezza le rispettive sfere giurisdizionali, preferendo muoversi tra ambiguità, sotterfugi e compromessi. Significativa in tal senso è la controversia sorta attorno alla "pubblicazione" dell'indice clementino (1596), che la Repubblica di Venezia non accetta nei suoi territori se non dopo lunghe trattative con Roma, concluse con un concordato e un compromesso sulle forme della sua pubblicizzazione. O ancora - per citare solo alcuni episodi - le trattative tra Venezia e Roma relative a proibizioni emanate dalla Congregazione dell'Indice dopo la promulgazione dell'indice del 1596; le mediazioni sul controllo preventivo di scritti atti a suscitare perplessità da parte ecclesiastica o secolare e le tortuose vicende editoriali attraverso cui si arriva alla loro stampa, come nel caso dei Saggi morali di Francis Bacon²2; gli escamotages per sottrarre ai controlli opere controverse, come l'uso di falsi dati bibliografici.

Concentrandosi sulle modalità con cui le autorità si prefiggono di ottenere il controllo esclusivo sulla comunicazione, Infelise circoscrive il problema del controllo dei libri soprattutto ai Riformatori dello Studio di Padova, all'inquisitore e al nunzio apostolico, lasciando in ombra il ruolo non irrilevante svolto dalle Congregazioni dell'Indice e dell'Inquisizione. Se negli anni a cavallo tra Cinquecento e Seicento la Repubblica è fortemente impegnata a contrastare l'intervento delle autorità ecclesiastiche, che dal canto loro possono avvalersi anche di confessori e predicatori come efficaci strumenti di persuasione, dopo la morte di Paolo Sarpi (15 gennaio 1623) il progetto di porre la stampa al servizio del rafforzamento dello Stato è ormai naufragato silenziosamente. Le esigenze di politica internazionale (guerra dei 30 anni), la necessità dell'appoggio di Roma (guerra di Candia), i conflitti interni al patriziato inducono la Repubblica a rinunciare a nuovi contenziosi con la Sede Apostolica. Sotto questo profilo è emblematica la sorte degli scritti di Sarpi ${ }^{23}$, che hanno una straordinaria circolazione in Europa, ma non vengono pubblicati a Venezia fino al 1776

\footnotetext{
${ }^{22}$ INFELISE, Mario, I padroni dei libri, cit., p. 69.

${ }^{23}$ Ibidem, 85-124.
} 
se non con qualche mascheramento che ne cela l'origine veneziana. La stretta vigilanza ecclesiastica sulle opere del servita induce del resto Infelise, nonostante il proposito di ridimensionarne il peso, ad affermare che «il cordone sanitario allestito intorno all'Italia qualche efficacia dovette averla avuta» 24 .

Così, l'intransigenza con cui la questione della stampa viene affrontata dal doge Leonardo Donà e dal frate servita si stempera «nell'azione quotidiana degli inquisitori e degli uffici di censura della Repubblica, sulla spinta di esigenze dettate di volta in volta dalle convenienze delle parti»25 (p. 85). Ormai lontani gli scontri giurisdizionali di inizio Seicento, la Repubblica ripiega sull'invettiva «anticlericale, violenta, anche blasfema, ma senza prospettive e progetto ${ }^{26}$ contro Urbano VIII. Rari sono, in effetti, i momenti di fiera reazione alle intromissioni ecclesiastiche - per esempio negli anni Quaranta, quando la produzione libertina, legata ai membri dell'Accademia degli Incogniti, per la sua dimensione anticuriale diviene il punto di riferimento culturale del patriziato veneziano, nonché il nucleo principale della produzione dei torchi veneziani, in un periodo in cui la produzione editoriale attraversa una fase recessiva.

Non migliore appare il bilancio del tribunale inquisitoriale e dei tribunali vescovili, $\mathrm{i}$ cui uomini, nonostante la lunga esperienza nella gestione ordinaria dei controlli «di fronte all'impossibilità di vigilare sugli scritti e sulla lettura, in mancanza di strumenti che per funzionare avrebbero dovuto avere una copertura europea, si accontentano di una minuta azione poliziesca sugli elementi più deboli della catena sociale e su aspetti tutto sommato secondari, come i manoscritti di magia» ${ }^{27}$. Non vi è dubbio, poi, che obiettive difficoltà rendono la loro azione tutt'altro che lineare e incontrastata: la capillare diffusione dei testi di magia in tutte le classi sociali; la circolazione clandestina di manoscritti; gli stratagemmi di librai e tipografi, restii a rinunciare ai profitti, quali edizioni con note tipografiche falsificate, stampe all'estero con falsi luoghi di edizione ${ }^{28}$. Nonostante i limiti ravvisati nell'azione censoria delle autorità ecclesiastiche e civili, dalle dense pagine di Infelise risulta così scalfita l'immagine idealizzata di Venezia proposta nelle Guerre culturali di Muir, che ne fa negli anni tra il 1590 e il 1660 la sede di una libera e fiorente industria editoriale, che pubblica libri senza interferenze di censure governative o ecclesiastiche ${ }^{29}$. Come l'autorità ecclesiastica, anche quella civile

\footnotetext{
24 Ibidem, p. 98.

25 Ibidem, p. 85.

${ }^{26}$ Ibidem, p. 130.

27 Ibidem, p. 204.

${ }^{28} \mathrm{Su}$ questi temi, cfr. BARBIERATO, Federico, Nella stanza dei circoli. Clavicula Salomonis e libri di magia a Venezia nei secoli XVII e XVIII, Milano, Sylvestre Bonnard, 2002.

29 MUIR, Edward, Guerre culturali. Libertinismo e religione alla fine del Rinascimento, RomaBari, Laterza, 2008.
} 
agisce nella consapevolezza che proibizione di libri e censura possono contribuire al rafforzamento dei propri poteri. Infelise precisa inoltre come siano indubbie le differenze tra Venezia e gli altri Stati italiani dove le istituzioni ecclesiastiche non incontrano ostacoli e con il resto d'Europa «dove si dovette attendere la seconda metà del Settecento per veder riprendere con maggiore decisione quella politica giurisdizionale che si era provato ad avviare oltre 150 anni prima»30. Tali differenze meriterebbero, peraltro, di essere approfondite sia per quanto riguarda alcuni Stati della penisola, come la Savoia e il Regno di Napoli sia per quanto riguarda l'Europa cattolica, dove, per esempio, la Francia e persino la cattolicissima Spagna non placitarono mai gli indici romani ${ }^{31}$.

La scelta di analizzare entrambi i testi si giustifica con la volontà di adottare una messa a fuoco che, partendo dallo studio del singolo episodio, approfondisce l'ampio conflitto tra Stato e Chiesa, conflitto in cui la molteplicità dei casi esaminati invita a studiare la censura in relazione ai contesti in cui si cala, cioè a una realtà fluida e complessa che forse può - ed è un punto che meriterebbe di essere approfondito influire sulle decisioni centrali.

A suggerire una lettura collettiva è anche la comune scelta degli autori di analizzare il potere nell'ottica di tutti i soggetti coinvolti: chi lo gestisce, ma anche chi tenta di emanciparsi dalla duplice tutela ecclesiastica e civile. Ecco allora che al centro dei due libri non ci sono soltanto le autorità e le forme della comunicazione "dall'alto". Se il merito di de Vivo risiede soprattutto nell'avere individuato il terreno di incontro e di scontro tra le pratiche dell'oralità e della scrittura e quello di Infelise della stampa e della censura, il merito di entrambi consiste nell'aver portato alla ribalta vicende di uomini. Non solo patrizi e autorevoli ecclesiastici - tra cui primeggia Paolo Sarpi, figura chiave dei volumi, attorno a cui ruota il problema teorico sollevato ad ogni conflitto: alla Chiesa il controllo dei libri teologici, allo Stato quello sugli altri testi -, ma anche scrittori, come il canonico lateranense Apollinare Calderini da Ravenna, la cui vicenda - i suoi Discorsi sopra la ragion di Stato del Botero, critici verso il sistema politico e la storia della Repubblica degli ultimi due secoli, cadono nelle mire del Consiglio dei Dieci - anima le prime pagine di I padroni dei libri. Grandi stampatori e, dopo il 1630, piccoli tipografi che, con il tracollo della produzione editoriale veneziana anche in conseguenza del crollo demografico, vivono di espedienti, sono incapaci di

\footnotetext{
${ }^{30}$ INFELISE, Mario, I padroni dei libri, cit., p. 205.

${ }^{31}$ FRAGNITO, Gigliola, «Diplomazia pontificia e censura ecclesiastica durante il regno di Enrico IV», in Rinascimento, 42, 2002, pp. 143-167; ID., Per una geografia delle traduzioni bibliche nell'Europa cattolica (sedicesimo e diciassettesimo secolo), in QUANTIN, Jean-Louis, WAQUET, Jean (sous la dir. de), Papes, princes et savants dans l'Europe moderne. Mélanges à la mémoire de Bruno Neveu, Genève, Librairie Droz, 2007, pp. 51-77.
} 
innovarsi; professionisti dell'informazione, avventurosi librai, come Roberto Meietti, che durante l'Interdetto riceve le maggiori commissioni della Repubblica, descritto da Infelise al centro dei traffici clandestini tra Venezia e l'Europa per più di mezzo secolo (dal 1580 al 1640).

Infine, conforta una lettura unitaria dei testi l'identico cronotopo: la Serenissima del secolo XVII,città in cui il tema della comunicazione politica e del controllo delle informazioni è all'ordine del giorno presso una classe dirigente gelosa della segretezza, ma costretta a confrontarsi con le allarmanti conseguenze dell'emergere a tutti i livelli sociali di curiosità nei confronti della politica e della religione, anche i barbieri, gli spezieri, i merciai protagonisti delle pagine di de Vivo prendono parte a discussioni sulle "cose del mondo". Discussioni - altro punto in comune - che nascono nella concretezza delle botteghe, siano quelle di barbieri (de Vivo) o di librai (Infelise) alle prese con i tentativi di aggirare divieti economicamente deprimenti per l'editoria. Discussioni legate alla diversa possibilità di accesso alle informazioni e alla capacità di elaborarle, nutrite di resoconti orali e scritti, di informazioni desunte da relazioni, gazzette, fogli volanti. Discussioni che testimoniano l'esistenza di un'embrionale opinione pubblica, priva di quella cultura associata all'idealtipica società borghese habermasiana e priva di quella forza necessaria per esprimersi in maniera aggressiva nella forma della rivolta contro il potere costituito, come nella Napoli di Masaniello (1648), ma con cui non può non confrontarsi l'azione delle censure. Azione che sarebbe interessante approfondire non solo in un contesto unicamente urbano, quale quello scelto dai due autori: quali notizie relative all'esperienza veneziana giungevano nelle zone limitrofe ${ }^{2}$ ? Come venivano recepite, interpretate, modificate ed eventualmente "soffocate" dal potere? E infine: come influisce un contesto non urbano sulla formazione di una "proto-opinione" pubblica?

\footnotetext{
${ }^{32}$ Su cui FLORIO, Giovanni, Rappresentanti e rappresentazioni delle comunità di Terraferma nella Venezia dell'Interdetto (1606-1607),Tesi di dottorato in Storia sociale europea dal Medioevo all'Età contemporanea - Università degli Studi di Venezia, Venezia, a.a. 2012/2013.
} 


\section{* L'autore}

Silvia Manzi è laureata in Lettere classiche (Università degli Studi di Parma), diplomata in Archivistica, Paleografia, Diplomatica (Archivio di Stato di Modena) e attualmente dottoranda (Università degli Studi di Teramo). Il suo progetto di ricerca riguarda la volgarizzazione delle bolle pontificie durante la Controriforma. I suoi interessi accademici vertono sugli usi del latino e del volgare nella comunicazione della Chiesa cattolica, sulla storia della censura ecclesiastica e dell'Inquisizione, sui movimenti ereticali nella prima età moderna.

URL: < http://www.studistorici.com/progett/autori/\#Manzi >

\section{Per citare questo articolo:}

MANZI, Silvia, «Comunicazione politica, informazione e censura a Venezia nella prima età moderna. RECENSIONI: Filippo DE VIVO, Patrizi, informatori, barbieri. Politica e comunicazione a Venezia, Milano, Feltrinelli, 2012, 466 pp.; Mario INFELISE, I padroni dei libri. II controllo sulla stampa nella prima età moderna, Roma-Bari, Laterza, 2014, 226 pp.», Diacronie. Studi di Storia Contemporanea : "Se creare è definire", 29/03/2016,

URL:<http://www.studistorici.com/2016/03/29/manzi_numero_25/ >

\section{Diacronie Studi di Storia Contemporanea 0 www.diacronie.it}

Risorsa digitale indipendente a carattere storiografico. Uscita trimestrale. redazione.diacronie@hotmail.it

Comitato di redazione: Jacopo Bassi - Luca Bufarale - Elisa Grandi - Antonio César Moreno Cantano - Deborah Paci - Fausto Pietrancosta - Alessandro Salvador - Matteo Tomasoni - Luca Zuccolo

Diritti: gli articoli di Diacronie. Studi di Storia Contemporanea sono pubblicati sotto licenza Creative Commons 3.0. Possono essere riprodotti e modificati a patto di indicare eventuali modifiche dei contenuti, di riconoscere la paternità dell'opera e di condividerla allo stesso modo. La citazione di estratti è comunque sempre autorizzata, nei limiti previsti dalla legge. 